\title{
Erratum: Energy nonequipartition in gas mixtures of inelastic rough hard spheres: The tracer limit [Phys. Rev. E 96, 052901 (2017)]
}

\author{
Francisco Vega Reyes, Antonio Lasanta, Andrés Santos •, and Vicente Garzó
}

(Received 17 September 2019; published 1 October 2019)

DOI: 10.1103/PhysRevE.100.049901

We have recently noted a small bug in the molecular dynamics (MD) code employed in the published version of the paper. Once corrected, the agreement between $\mathrm{MD}$ and theoretical results is much better than reported. Note, however, that, in a follow-up paper [1], the MD code was the correct one.

The list of needed changes is as follows:

(1) Figures 2-4 of the paper should be replaced by Figs. 1-3 of this Erratum.

(2) In the caption of Fig. 3, the sentence

"Except for the MD data in panel (b), the error bars in the simulation data are smaller than the size of the symbols." should be replaced by

"The error bars in the simulation data are smaller than the size of the symbols."

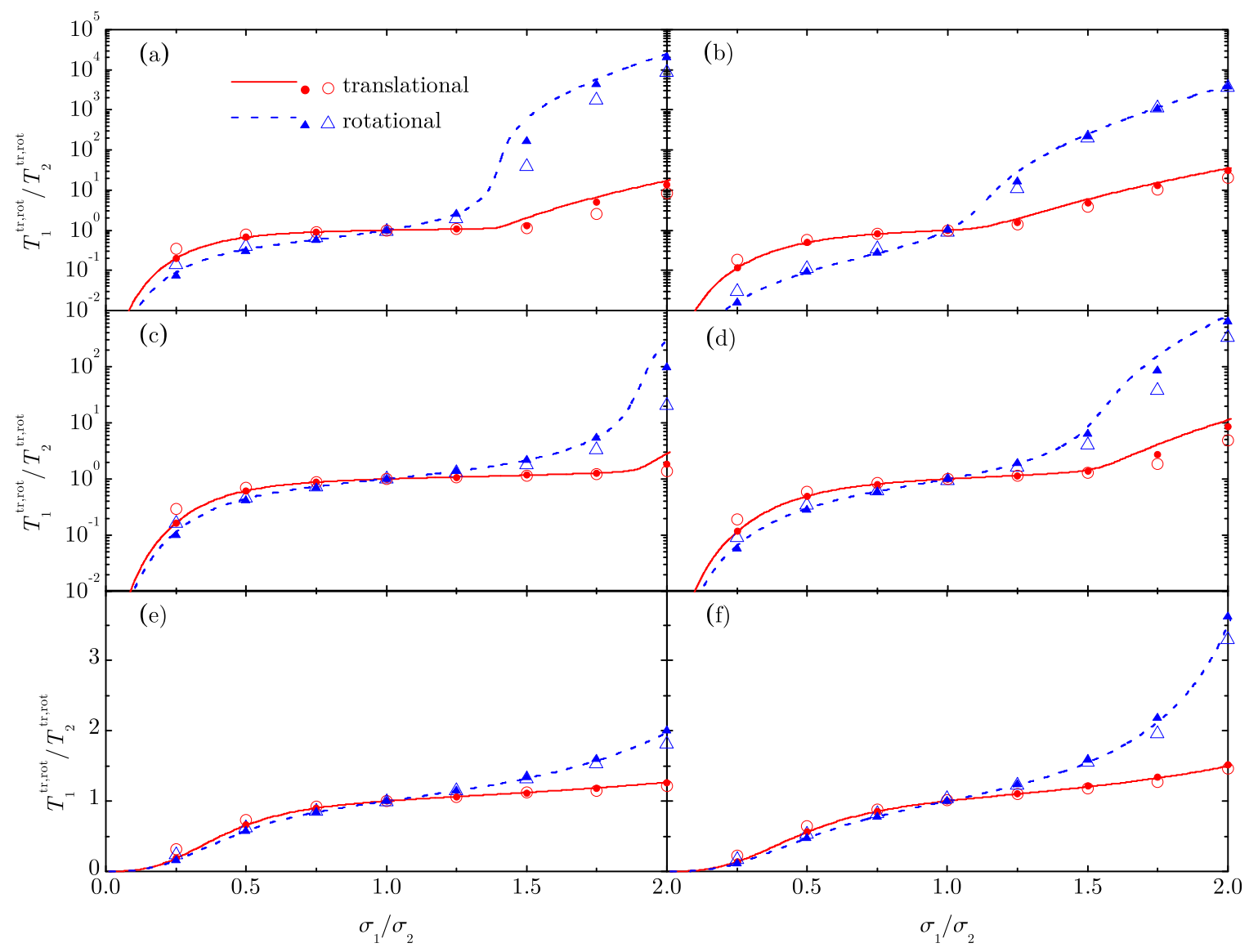

FIG. 1. Plot of the temperature ratios $T_{1}^{\mathrm{tr}} / T_{2}^{\mathrm{tr}}$ (solid lines and circles) and $T_{1}^{\mathrm{rot}} / T_{2}^{\mathrm{rot}}$ (dashed lines and triangles) versus $\sigma_{1} / \sigma_{2}$ for systems (a) A, (b) B, (c) C, (d) D, (e) E, and (f) F (see Table II of the original paper). The lines are theoretical predictions, the filled symbols are DSMC results, and the open symbols are MD results. The error bars in the simulation data are smaller than the size of the symbols. Note the vertical logarithmic scales in panels (a)-(d). 

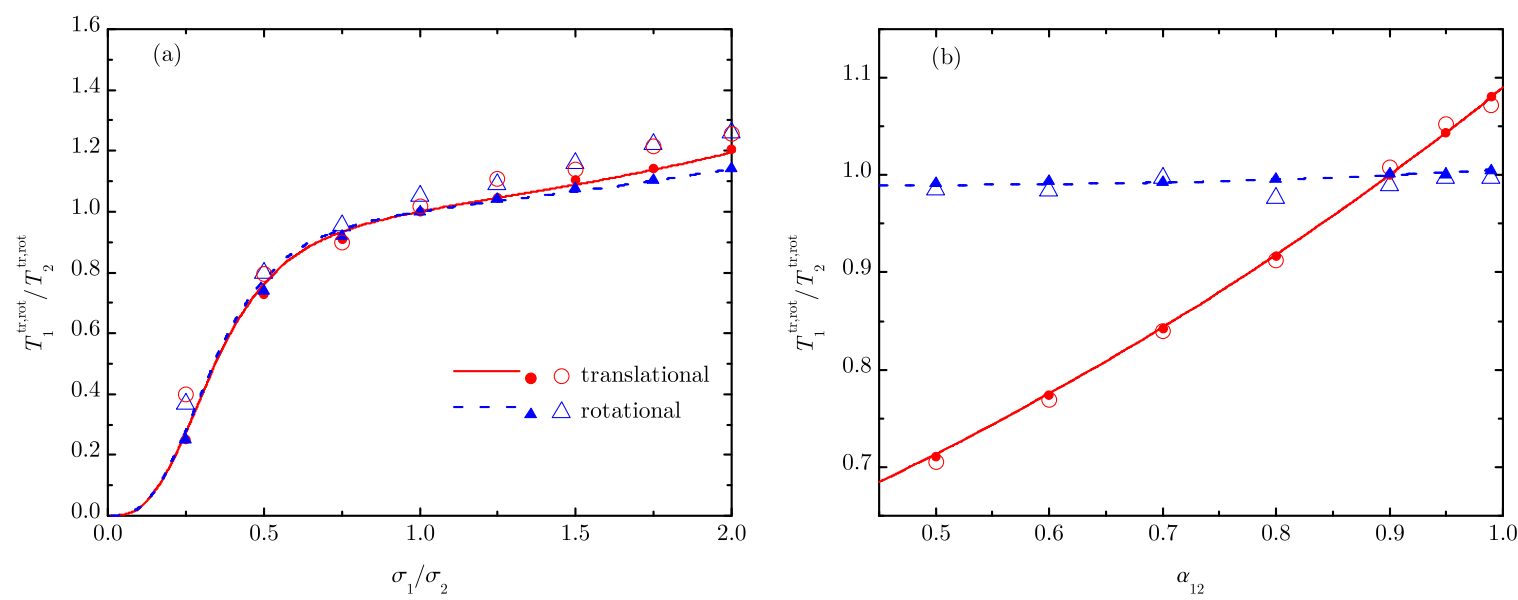

FIG. 2. Plot of the temperature ratios $T_{1}^{\mathrm{tr}} / T_{2}^{\mathrm{tr}}$ (solid lines and circles) and $T_{1}^{\text {rot }} / T_{2}^{\text {rot }}$ (dashed lines and triangles) versus (a) $\sigma_{1} / \sigma_{2}$ and (b) $\alpha_{12}$ for systems (a) G and (b) H (see Table II of the original paper). The lines are theoretical predictions, the filled symbols are DSMC results, and the open symbols are MD results. The error bars in the simulation data are smaller than the size of the symbols.

(3) In Sec. IV, the sentences

"The MD data have been restricted to $\sigma_{1} / \sigma_{2} \leqslant 1.5$ because we have observed that for $\sigma_{1} / \sigma_{2} \gtrsim 1.25$ the tracer particle is massive enough to disturb the state of the host gas so that the Boltzmann-Lorentz treatment is not applicable. This does not happen in the direct simulation Monte Carlo (DSMC) simulations since in that case one numerically solves Eqs. (18) independently."

should be removed.

(4) In Sec. IV, the sentence

"An exception is the rotational temperature ratio $T_{1}^{\text {rot }} / T_{2}^{\text {rot }}$ at $\sigma_{1} / \sigma_{2}=1.5$, where the MD data are clearly larger than both the theoretical and DSMC values."

should be replaced by

"An exception is the rotational temperature ratio $T_{1}^{\text {rot }} / T_{2}^{\text {rot }}$ at $\sigma_{1} / \sigma_{2} \geqslant 1.5$ where the MD data are typically smaller than both the theoretical and the DSMC values."

(5) In Sec. IV, the sentence

"The theory agrees quantitatively well with the DSMC data but only qualitatively with the MD simulations." should be replaced by

"The theory agrees quantitatively well with the DSMC data and semiquantitatively with the MD simulations."

(6) In Sec. IV, the sentence

"The agreement between theory and simulations is very good, although the fluctuations in the MD data for $T_{1}^{\text {rot }} / T_{2}^{\text {rot }}$ are noticeable."

should be replaced by

"The agreement between theory and simulations is very good."
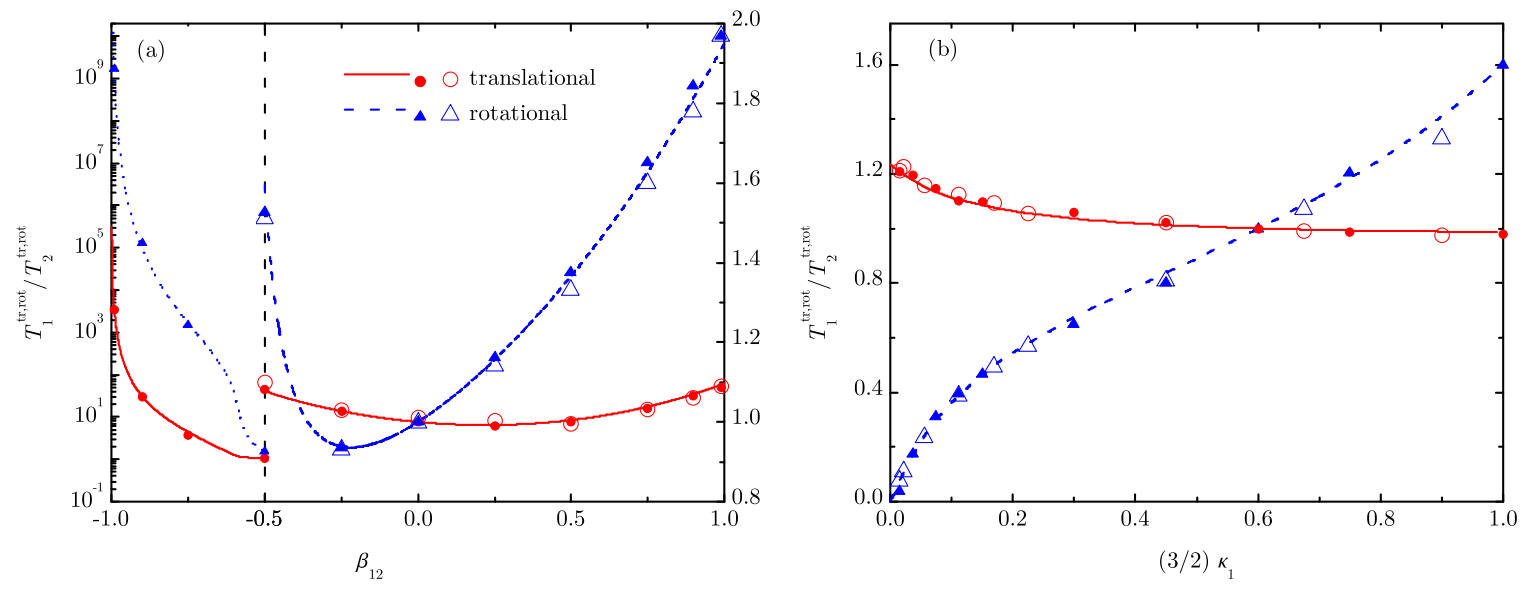

FIG. 3. Plot of the temperature ratios $T_{1}^{\mathrm{tr}} / T_{2}^{\mathrm{tr}}$ (solid lines and circles) and $T_{1}^{\text {rot }} / T_{2}^{\text {rot }}$ (dashed lines and triangles) versus (a) $\beta_{12}$ and (b) $\frac{3}{2} \kappa_{1}$ for systems (a) I and (b) J (see Table II of the original paper). The lines are theoretical predictions, the filled symbols are DSMC results, and the open symbols are MD results. The error bars in the simulation data are smaller than the size of the symbols. Note that, in panel (a), the vertical scale is logarithmic for $-1 \leqslant \beta_{12} \leqslant-0.5$ and normal for $-0.5 \leqslant \beta_{12} \leqslant 1$. 
(7) In Sec. V, the sentences

"We have observed that limitations of the molecular chaos ansatz are relevant only for large tracer particles and/or very inelastic gases, as shown in Figs. 2 and 3(a). Even in those cases, the theoretical description agrees qualitatively well with MD simulations."

should be replaced by

"We have observed that limitations of the molecular chaos ansatz are noticeable only for large tracer particles and/or very inelastic gases as shown in Figs. 2 and 3(a). Even in those cases, the theoretical description agrees semiquantitatively well with MD simulations."

An integrated version of the paper, including the above modifications, is available in Ref. [2].

[1] A. Lasanta, F. Vega Reyes, V. Garzó, and A. Santos, Intruders in disguise: Mimicry effect in granular gases, Phys. Fluids 31, 063306 (2019).

[2] F. Vega Reyes, A. Lasanta, A. Santos, and V. Garzó, arXiv:1709.00242v3. 